\title{
Global perspectives on operative vaginal deliveries
}

\author{
Vannevel $\mathbf{V}^{\mathbf{1}}$, Swanepoel $\mathbf{C}^{\mathbf{1}}$, Pattinson $\mathbf{R C}^{\mathbf{1}}$ \\ ${ }^{1}$ South African Medical Research Council/University of Pretoria, Maternal and Infant \\ Health Strategies Unit; Department of Obstetrics and Gynaecology, University of \\ Pretoria, South Africa
}

\section{Corresponding author}

Valerie Vannevel

valerie.vannevel@up.ac.za

valerie.vannevel@gmail.com

+27123731078

+27729988704 


\begin{abstract}
Operative vaginal delivery (OVD) refers to the use of an instrument (forceps or vacuum device), to assist with delivery of the fetus from the vagina. This can help improve maternal and fetal outcomes and has to be weighed up against the risks and benefits of performing second stage caesarean deliveries. OVD forms an integral part of basic emergency obstetric care and a skilled birth attendant's duties. Outlet forceps and vacuum extraction should be used to shorten the second stage of labour and to improve maternal and fetal outcomes associated with delayed second stage. Despite the known benefit of OVD, available data on the use of operative vaginal deliveries in low- and middle-income countries show very low rates, mostly due to lack of skilled healthcare workers and equipment shortages. Increased use of OVD can safely reduce the number of second-stage caesarean deliveries with its associated morbidity and mortality. We recommend implementing training programs to increase the number of skilled healthcare workers and strengthening health systems to provide birthing facilities with the equipment required to perform OVD.
\end{abstract}

Keywords: operative vaginal delivery; vacuum; forceps; low- and middle-income countries 


\section{INTRODUCTION AND BACKGROUND}

Operative vaginal delivery (OVD) refers to the use of an instrument (forceps or vacuum device), to assist with delivery of the fetus from the vagina. This can be achieved with or without maternal pushing. The decision to perform an assisted delivery can improve maternal and fetal outcomes and has to be weighed up against the risks and benefits of performing caesarean delivery during the second stage of labour. Indications for performing an OVD include fetal distress, delay in the second stage or maternal contraindications to Valsalva.

The fifth Millennium Development Goal established reducing maternal mortality as a priority for the international community [1]. More than half of the estimated 536000 global annual maternal deaths occur in Sub-Saharan Africa. In certain regions, the maternal mortality ratio exceeds 900 per 100000 live births [2,3]. Maximizing intrapartum care for pregnant women seems to be the most important intervention in reducing the high maternal mortality rate. Around $70 \%$ of deaths are due to haemorrhage, hypertensive diseases in pregnancy, sepsis, unsafe abortion and obstructed labour [4].

The World Health Organization (WHO) defines seven basic services as prerequisites for emergency obstetric care [5]:

-Parenteral antibiotic administration

-Use of uterotonic drugs

-Magnesium sulfate administration to women with preeclampsia/eclampsia 
-Manual removal of the placenta

- Removal of retained products of conception with the use of vacuum aspiration or by dilation and curettage

-Performing assisted vaginal delivery with the use of forceps or vacuum extraction -Performing basic neonatal resuscitation with a bag and mask

Assisted delivery rates vary greatly between settings and the ideal rate is not known. In developed countries, the rates vary: from $10-15 \%$ in the UK to around $4.5 \%$ in the USA where its rate has halved in the last 20 years [6,7]. Lower rates are expected in lower resource settings because of the higher mean parity, the unavailability of epidural analgesia and electronic fetal monitoring as well as lack of facilities providing OVD. The rate of OVD is also inversely proportional to the caesarean delivery rate. This leads to an unmet need and higher incidence of complications of obstructed second stage of labour such as stillbirths, uterine rupture and vesicovaginal fistulas.

\section{FISTULAE, SPHINCTER INJURIES AND FEMALE GENITAL MUTILATION}

In low- and middle-income countries, especially in sub-Saharan Africa, vesicovaginal fistulae are more common than in high-income countries. Obstructed labour caused by cephalopelvic disproportion (CPD) and prolonged second stage of labour can cause maternal injuries leading to vesicovaginal fistulae. Obstetric fistulae are a direct consequence of obstructed labour. The permanent leakage of urine and/or faeces through the vagina can lead to the rejection of these women resulting in isolation. The MOMA study was a multi-centre, population-based prospective study, carried out in six major 
West African countries. Over 20000 women were followed up during pregnancy and after delivery. Two women were diagnosed with a vesicovaginal fistula after delivery. The incidence rate was 10.3 per 100000 deliveries and 0 per 100000 in major cities [8]. In the rural area involved in this study, the incidence of obstetric fistulae is around 123.9 per 100000 deliveries, which is much higher than previously thought [9]. These data stress the importance of timely diagnosis and intervention of obstructed labour and provision of good quality emergency obstetric care including OVD.

Obstetric anal sphincter injury (OASIS) is a major cause of maternal morbidity and is a risk factor for the development of fistulae and faecal incontinence. Ramm et al showed that OVD was complicated by OASIS in $24 \%$ of vacuum deliveries compared to $4 \%$ in normal vaginal deliveries [10]. Data regarding the greater risk of forceps OVD than vacuum OVD for OASIS are conflicting, but seem to show more OASIS with forceps than with vacuum. Hehir et al looked at vaginal deliveries at two large obstetric hospitals during an eight year period [11]. Out of 100307 vaginal deliveries there were 2121 cases of OASIS (2.1\% incidence): OASIS was more common with forceps delivery than NVD (8.6\% versus $1.3 \%, \mathrm{p}<0.0001$, OR: 7.1, CI: 6.4-7.9). Vacuum delivery also carried an increased risk of OASIS (3.7\% versus $1.3 \%, \mathrm{p}<0.0001$, OR: 2.9 , CI: $2-2.6)$. The Cochrane review on choice of instruments for assisted vaginal delivery mentions that sphincter injuries (third- and fourth-degree perineal tears) are more likely with forceps than vacuum [12].

The WHO describes female genital mutilation (FGM) as all procedures that intentionally alter or cause injury to the female genital organs for non-medical reasons (such as partial or total removal of the external female genitalia or other injuries to the female genital 
organs) [13]. FGM is classified into four types and is prevalent among some ethnic groups, concentrated in Africa, the Middle East and Asia. FGM is usually performed as a part of culture and tradition and carries no health benefit for the girl or woman. Type III FGM, also referred to as infibulation, is the narrowing of the vaginal opening through the creation of a covering seal. The seal is formed by cutting and repositioning the labia minora, or labia majora, sometimes through stitching, with or without removal of the clitoris (clitoridectomy). Deinfibulation refers to the practice of cutting open the sealed vaginal opening, which may be necessary to facilitate childbirth. Reinfibulation (restitching after deinfibulation) is not recommended.

FGM can lead to obstructed labour and is associated with an increased risk of OVD and perineal trauma. Therefore, given the causal relationship between obstructed labour and fistulas, FGM may lead to a higher risk of developing fistulas after delivery. Abdulcadir et al performed a retrospective analysis of women with FGM who attended a specialized clinic at a tertiary center in Switzerland [14]. They found an operative vaginal delivery rate of $22 \%$ in all deliveries of women with FGM and $20 \%$ in women with type III FGM, which is higher than the average in Switzerland.

\section{WORLDWIDE USE OF OVD}

Operative vaginal deliveries are more common in high-income countries than in low- and middle-income countries. The rate of OVD ranges from 10-15\% of all live births in the UK to as low as $1.5 \%$ in other countries. In the UK, the rates have remained fairly constant, but the preferred instrument has moved from forceps to vacuum extractor. Bailey et al assessed the incidence of operative vaginal deliveries in low and middle 
income countries [15]. They included hospital and non-hospital sites in 36 countries, 27 of which were located in Sub-Saharan Africa. They found that instrumental delivery was not widely practiced, but it was more likely to occur in hospitals than in clinics and health centers. Fifty-three percent of hospitals in Sub-Saharan Africa reported operative vaginal deliveries in the recent past compared to $6 \%$ of non-hospital sites. The total percentage of operative vaginal deliveries was around $1 \%$. The reasons cited for not performing OVD were inadequate training, equipment shortages and policies failing to support the use of OVD. Specialist obstetricians most often provided OVD in hospitals and midwives in non-hospital sites. Mozambique was an exception to the rule where midwives were trained in performing vacuum extraction as part of a government initiative.

\section{OVD RATES IN LMIC}

Nolens et al did a prospective study in Uganda including 13152 deliveries, including 358 vacuum extraction operative vaginal deliveries [16]. There were no maternal deaths reported in the vacuum extraction group, compared to five maternal deaths attributed to complications during or after second stage caesarean delivery. In terms of fetal outcome, the perinatal mortality rate was similar in the two groups $(8.4 \%$ in the OVD group vs $11 \%$ in the second stage caesarean delivery group). This study highlighted the improved maternal outcomes and similar perinatal outcomes when OVD is compared to second stage caesarean delivery. Their conclusion was that vacuum deliveries can help improve maternal outcomes and reduce complications related to second stage caesarean deliveries. 
Caesarean deliveries performed during the second stage of labour are associated with an increased risk of maternal morbidity, such as prolonged hospital stay, haemorrhage, risk of bladder trauma and unintended extensions of the uterine incision $[17,18]$. Several studies looking at perinatal outcomes after second-stage caesarean delivery compared to OVD found no difference or worse outcomes in the caesarean delivery group $[19,20]$.

There are limited data around the use of OVD in different countries and regions. Levine et al did a study looking at emergency obstetric care in Zambia [21]. In terms of OVD, only $6.1 \%$ of health centres had vacuum extractors and $9.1 \%$ of health centres had forceps available. Only $5.7 \%$ of healthcare workers felt comfortable performing assisted vaginal deliveries (forceps or vacuum). Adaji et al found an OVD rate of $3.6 \%$ with prevalence rates of $1.0-3 \%$ for forceps delivery and $1.4-1.5 \%$ for vacuum extraction in Nigeria [22]. The paper by Duysburgh et al reporting pre-intervention measurements of quality of routine antenatal and childbirth care in rural districts in Burkina Faso, Ghana and Tanzania showed that, even though quality scores for childbirth care were reasonably high, none of the sites performed OVD; equipment to perform OVD was absent in the sites [23]. A facility-based cross-sectional study reported by Echoka et al collected data on emergency obstetric care in 40 health facilities in Malindi District in Kenya [24].None of the assessed facilities provided OVD, neither by vacuum or forceps. Pattinson et al reported that the assisted delivery rate in South Africa (reported in 2010-2011) was $0.52 \%$ for vacuum and $0.15 \%$ for forceps whilst the caesarean delivery rate was $21 \%$ [25]. The study found a negative correlation between the use of OVD and perinatal deaths secondary to intrapartum asphyxia and trauma. They hypothesized that increasing 
the use of OVD in South Africa will likely help reduce perinatal morbidity and mortality due to intrapartum asphyxia. It is also thought to reduce maternal morbidity and mortality related to second stage caesarean deliveries. An increase in the use of OVD can be achieved through a unified effort by managers, educators, trainers and supervisors working in obstetrics.

Chang et al mention the scarcity of available data on use of vacuum extraction in South America [26]. Their prospective study in Ecuador looked at rising caesarean delivery rates which were linked to increasing costs and maternal morbidity. Operative vaginal delivery was not done routinely at their institution: they reported a $1.9 \%$ incidence of vacuum extraction in 1 hospital in Ecuador; the lack of skilled healthcare workers and absence of an established training program being the main reasons for the low rates. Vacuum delivery was shown to be a cost effective and safe alternative to caesarean delivery and the investigators suggested that adequate training programs in OVD can help reduce caesarean delivery rates with the associated complications thereof.

\section{CULTURAL BELIEFS AND HIV INFECTION}

We have found no data on cultural beliefs around OVD such as declining performing or undergoing assisted delivery for religious or cultural reasons. Healthcare workers might be hesitant to perform OVD in HIV-positive women. The Cochrane review on choice of instrument for assisted vaginal delivery and the RCOG guideline on OVD suggest a theoretically increased risk of mother-to-child transmission during OVD due to scalp injury; however, there are very little data available [6,12]. The National Study of HIV in 
Pregnancy and Childhood in the UK and Ireland collected data of 9072 live births in HIV-positive women since 2008 [27]. Operative vaginal delivery was performed in 251 women, 1 infant was reported to be HIV-positive. The RCOG states that blood-borne viral infections of the mother are no contra-indication to performing OVD but one should avoid scalp trauma.

\section{ANALGESIA AND OVD IN LMIC}

We did not find any data on the use of regional versus local analgesia for performing OVD in LMICs. In general, adequate analgesia is recommended. Epidural analgesia is often not available, as only trained doctors can perform the procedure and many (rural) birthing facilities only have midwives and nurses on site. For local anesthesia, local infiltration or a pudendal block can be used. However, in settings with high HIV prevalence, a pudendal block is not commonly performed due to the risk of needle pricks for the birth attendant.

\section{IMPROVED TRAINING CAN INCREASE OVD RATES}

Improved training in operative vaginal deliveries can help lead to increased rates of vacuum and forceps deliveries. The Advanced Life Support in Obstetrics (ALSO) provider course, a two day training course, was developed to teach birth attendants evidence-based management of emergencies in obstetrics. The goals are to improve patient care and to increase healthcare providers' skills level in emergency obstetric care. The results from Colombia, Guatemala, Honduras and Tanzania showed improved 
obstetric outcomes after the training has been completed [28]. Two hospitals in Colombia and Guatemala had a reduction in maternal mortality after implementation of the training. Vacuum delivery can help reduce the need for caesarean deliveries. The results from Honduras showed increased vacuum deliveries after introduction of ALSO. In Tanzania, assisted vaginal deliveries increased the year after introduction of ALSO. The results at 6 and 12 months showed that more than $90 \%$ of providers that utilized operative vaginal delivery felt that ALSO improved their skills as well as maternal and fetal outcomes. At the study hospital, vacuum deliveries were never done and forceps only rarely done before ALSO training. In contrast, 6 months after training, 13 (24.1\%) of the 54 participants had used a vacuum extractor and 18 (33.3\%) had used forceps. Twelve months after ALSO training, 18 (33.3\%) reported vacuum use and 20 (37.0\%) forceps use.

One concern to be raised might be the increased risk of failed OVD in birthing facilities without immediate access to caesarean delivery if OVD is more widely used. To avoid this complication, we suggest that birth attendants in remote facilities without theatre access should only perform OVD when the head is on the perineum (in case of suspected fetal compromise or failure to progress). Training programs should include topics such as assessing the position of the fetal head and assessing cephalo-pelvic disproportion to avoid further complications.

One clinical innovation, the Odon Device, is currently under development as a new instrument for OVD where a plastic sleeve is inserted around the fetus' head and a cuff is inflated to subsequently assist the operator in delivering the baby. One pilot clinical study was performed (and early terminated), the results suggest the Odon Device is feasible as 
a device to assist delivery [29]. This could be promising, especially for low- and middleincome countries. However, as this was a feasibility study where the device was applied in non-emergency conditions, more research is needed before this device can be implemented.

\section{CONCLUSION}

As shown above, OVD forms an integral part of basic emergency obstetric care and a skilled birth attendant's duties. Outlet forceps and vacuum extraction should be used to shorten the second stage of labour and to improve maternal and fetal outcomes associated with delayed second stage. Increased use of OVD can safely reduce the number of second-stage caesarean deliveries with its associated morbidity and mortality. We recommend the implementation of training programs in low and middle income countries for all skilled birth attendants to provide high-quality emergency obstetric care as well as support from health systems to provide all birthing facilities with the equipment required to perform OVD.

\section{CONFLICT OF INTEREST}

None.

\section{PRACTICE POINTS}

- The indications and benefits of operative vaginal deliveries are well established.

- The rates of operative vaginal deliveries are significantly lower in low and middle income countries than in high income countries. 
- Lack of skilled healthcare workers and lack of resources are the main reasons for the low rates of operative vaginal deliveries.

- To be able to provide operative vaginal deliveries at all birthing facilities as part of high-quality emergency obstetric care, training programs for all skilled birth attendants need to be established.

\section{RESEARCH AGENDA}

- Operative vaginal delivery and mother-to-child transmission of HIV

- Implementation strategies in low and middle income countries

\section{REFERENCES}

1. United Nations. United Nations Millennium Declaration. Resolution adapted by the General Assembly, 55th Session of the United Nations General Assembly, New York, 8 September 2000.

2. WHO, UNICEF, UNFPA, the World Bank. Maternal Mortality in 2005; estimates developed by WHO, UNICEF, UNFPA, and The World Bank. WHO press, Geneva, 2007. Available from http://www.who.int/whosis/mme_2005.pdf

3. Hill K, Thomas K, AbouZahr C, Walker N, Say L, Inoue M,et al. Estimates of maternal mortality worldwide between 1990 and 2005: an assessment of available data. Lancet 2007;370(9595):1311-9. 
4. Khan KS, Wojdyla D, Say L, Gülmezoglu AM, Van Look PF. WHO analysis of causes of maternal death: a systematic review. Lancet 2006;367(9516):1066-74. http://dx.doi.org/10.1016/s0140-6736(06)68397-9.

5. World Health Organization. Monitoring obstetric care: a handbook. WHO press, Geneva, 2009. Available from whqlibdoc.who.int/publications/2009/9789241547734_eng.pdf

6. Royal College of Obstetricians \& Gynaecologists. Operative Vaginal Delivery. London: RCOG, 2011. https://www.rcog.org.uk/en/guidelines-researchservices/guidelines/gtg26/ (accessed 25 August 2018).

7. Martin JA, Hamilton BE, Sutton PD, Ventura SJ, Menacker F, Kirmeyer S, et al. Births: Final data for 2006. National Vital Statistics Reports 2009; Volume 57, Number 7.

8. Vangeenderhuysen C, Prual A, Ould el Joud D. Obstetric fistulae: incidence estimates for sub-Saharan Africa. Int J Gynaecol Obstet 2001;73(1):65-6.

9. Prual A, Bouvier-Colle MH, de Bernis L, Bréart G. Severe maternal morbidity from direct obstetric causes in West Africa: incidence and case fatality rates. Bull World Health Organ 2000;78(5):593-602.

10. Ramm O, Woo VG, Hung YY, Chen HC, Ritterman Weintraub ML. Risk Factors for the Development of Obstetric Anal Sphincter Injuries in Modern Obstetric Practice. Obstet Gynecol 2018;131(2):290-6. http://dx.doi.org/10.1097/aog.0000000000002444.

11. Hehir MP, O'Connor HD, Higgins S, Robson MS, McAuliffe FM, Boylan PC et al. Obstetric anal sphincter injury, risk factors and method of delivery - an 8- 
year analysis across two tertiary referral centers. J Matern Fetal Neonatal Med. 2013;26(15):1514-6. http://dx.doi.org/10.3109/14767058.2013.791268.

12. O’Mahony F, Hofmeyr GJ, Menon V. Choice of instruments for assisted vaginal delivery. Cochrane Database of Systematic Reviews 2010, Issue 11. http://dx.doi.org/10.1002/14651858.cd005455.pub2.

13. World Health Organization [Internet]. Sexual and reproductive health: Female genital mutilation [cited 2018 Aug 24]. Available from: http://www.who.int/reproductivehealth/topics/fgm/health_consequences_fgm/en

14. Abdulcadir J, Dugerdil A, Yaron M, Irion O, Boulvain M. Obstetric care of women with female genital mutilation attending a specialized clinic in a tertiary center. Int J Gynecol Obstet 2016;132(2):174-8. http://dx.doi.org/10.1016/j.ijgo.2015.06.055.

15. Bailey PE, van Roosmalen J, Mola G, Evans C, de Bernis L, Dao B. Assisted vaginal delivery in low and middle income countries: an overview. BJOG 2017;124(9):1335-44. http://dx.doi.org/10.1111/1471-0528.14477.

16. Nolens B, Namiiro F, Lule J, van den Akker T, van Roosmalen J, Byamugisha J. Prospective cohort study comparing outcomes between vacuum extraction and second-stage cesarean delivery at a Ugandan tertiary referral hospital. Int J Gynecol Obstet 2018;142(1):28-36. http://dx.doi.org/10.1002/ijgo.12500

17. Allen VM, O'Connell CM, Baskett TF. Maternal and perinatal morbidity of caesarean delivery at full cervical dilatation compared with caesarean delivery in the first stage of labour. BJOG 2005;112(7):986-90. https://doi.org/10.1111/j.1471-0528.2005.00615.x. 
18. Murphy DJ, Liebling RE, Verity L, Swingler R, Patel R. Early maternal and neonatal morbidity associated with operative delivery in second stage of labour: A cohort study. Lancet 2001;358(9289):1203-7. https://doi.org/10.1016/s01406736(01)06341-3.

19. Werner EF, Janevic TM, Illuzzi J, Funai EF, Savitz DA, Lipkind HS. Mode of delivery in nulliparous women and neonatal intracranial injury. Obstet Gynecol 2011;118(6):1239-46. https://doi.org/10.1097/aog.0b013e31823835d3

20. Towner D, Castro MA, Eby-Wilkens E, Gilbert WM. Effect of mode of delivery in nulliparous women on neonatal intracranial injury. N Engl J Med 1999;341(23):1709-14. https://doi.org/10.1056/ nejm199912023412301.

21. Levine AC, Marsh RH, Nelson SW, Tyer-Viola L, Burke TF. Measuring access to emergency obstetric care in rural Zambia. Int J Emerg Med 2008;1(2):113-9. http://dx.doi.org/10.1007/s12245-008-0032-4.

22. Adaji SE, Shittu SO, Sule ST. Operative vaginal deliveries in Zaria, Nigeria. Ann Afr Med 2009;8(2):95-9. http://dx.doi.org/10.4103/1596-3519.56236.

23. Duysburgh E, Zhang WH, Ye M, Williams A, Massawe S, Sié A, et al. Quality of antenatal and childbirth care in selected rural health facilities in Burkina Faso, Ghana and Tanzania: similar finding. Trop Med Int Health 2013;18(5):534-47. http://dx.doi.org/10.1111/tmi.12076.

24. Echoka E, Kombe Y, Dobourg D, Makokha A, Evien-Olsen B, Mwangi M, et al. Existence and functionality of emergency obstetric care services at district level in Kenya: theoretical coverage versus reality. BMC Health Serv Res 2013;13:113. https://doi.org/10.1186/1472-6963-13-113. 
25. Pattinson RC, Vannevel V, Barnard D, Baloyi S, Gebhardt GS, le Roux K, et al. Failure to perform assisted deliveries is resulting in an increased neonatal and maternal morbidity and mortality: An expert opinion. S Afr Med J 2018;108(2):75-8. http://dx.doi.org/10.7196/samj.2017.v108i2.12786.

26. Chang X, Chedraui P, Ross MG, Hidalgo L, Afiel JP. Vacuum assisted delivery in Ecuador for prolonged second stage of labor: maternal-neonatal outcome. J Matern Fetal Neonatal Med 2007;20(5):381-4. http://dx.doi.org/10.1080/14767050701227927.

27. Peters H, Francis K, Harding K, Tookey PA, Thorne C. Operative vaginal delivery and invasive procedures in pregnancy among women living with HIV. Eur J Obstet Gynecol Reprod Biol 2017;210:295-9. http://dx.doi.org/10.1016/j.ejogrb.2016.12.016.

28. Dresang LT, González MM, Beasley J, Bustillo MC, Damos J, Deutchman M, et al. The impact of Advanced Life Support in Obstetrics (ALSO) training in low-resource countries. Int J Gynecol Obstet 2015;131(2):209-15. http://dx.doi.org/10.1016/j.ijgo.

29. Schvartzman JA, Krupitzki H, Merialdi M, Betrán AP, Requejo J, Nugyen MH, et al. Odon device for instrumental vaginal deliveries: results of a medical device pilot clinical study. Reprod Health 2018;15(1):45. http://dx.doi.org/10.1186/s12978-018-0485-8. 